\title{
The Ratchet Effect
}

\section{Dramatic and Sustained Changes in Health Care Utilization Following Admission to Hospital With Chronic Disease}

\author{
David Whyatt, PhD, * Raji Tenneti, PhD,* Julie Marsh, PhD,*+Sarah E. Bolt, BSc (Hons),* \\ Anna Kemp, PhD, $\ddagger \$$ Laura Firth, BSc (Hons), $\dagger$ Kevin Murray, MSc, $\dagger$ Berwin Turlach, PhD, $\dagger$ \\ and Alistair Vickery, MBBS, FRACGP*
}

\begin{abstract}
Objective: To describe the previously unexamined association between admissions to hospital with chronic disease and changes in all-cause health service utilization over time.
\end{abstract}

Research Design: A cohort study examining the population of Western Australia with hospitalizations for chronic disease from 2002 to 2010. A "rolling" clearance period is used to define "cardinal events," that is, a disease-specific diagnosis upon hospital admission, where such an event has not occurred in the previous 2 years. Changes in the rate of cardinal events associated with diagnoses of heart failure, type 2 diabetes, chronic obstructive pulmonary disease, cataract with diabetes, asthma, and dialysis are examined. Health service utilization (defined as inpatient days or emergency department presentations) 6 years preceding and 4 years following such events is presented.

Results: Cardinal events make up $40 \%-60 \%$ of all chronic disease admissions. A previously undescribed ratchet effect following cardinal events specifically associated with type 2 diabetes, heart failure, and chronic obstructive pulmonary disease is observed. This involves a 2- to 3-fold increase in inpatient days and emergency department presentations that are sustained for at least 4 years.

From the *School of Primary, Aboriginal and Rural Health Care, Faculty of Medicine, Dentistry and Health Sciences; $†$ School of Mathematics and Statistics, Faculty of Engineering, Computing and Mathematics; $¥$ School of Population Health, Faculty of Medicine, Dentistry and Health Sciences, University of Western Australia, Crawley, WA; and §Illawarra Health and Medical Research Institute, University of Wollongong, Wollongong, NSW, Australia.

Supported by the Department of Health Western Australia.

The authors declare no conflict of interest.

Reprints: David Whyatt, PhD, School of Primary, Aboriginal and Rural Health Care, Faculty of Medicine, Dentistry and Health Sciences, The University of Western Australia, M706, 35 Stirling Highway, Crawley, WA 6009, Australia. E-mail: david.whyatt@uwa.edu.au.

Supplemental Digital Content is available for this article. Direct URL citations appear in the printed text and are provided in the HTML and PDF versions of this article on the journal's Website, www.lww-medical care.com.

Copyright $(\mathbb{C} 2014$ by Lippincott Williams \& Wilkins. This is an open-access article distributed under the terms of the Creative Commons AttributionNonCommercial-NoDerivitives 3.0 License, where it is permissible to download and share the work provided it is properly cited. The work cannot be changed in any way or used commercially.

ISSN: 0025-7079/14/5210-0901
Conclusions: Cardinal events represent an important reference point to understand the impact of chronic disease on health service utilization. Events that herald such a marked transition in health service demand have not been previously described.

Key Words: chronic disease, longitudinal analysis, heart failure, type 2 diabetes, chronic obstructive pulmonary disease

(Med Care 2014;52: 901-908)

$\mathrm{H}$ ealth systems are under pressure from increasing incidence of chronic disease. ${ }^{1,2}$ Chronic conditions are prevalent in older populations, with $70 \%-80 \%$ of people aged 65 or over having at least 1 chronic condition. ${ }^{3}$ Chronic diseases, including type 2 diabetes mellitus, heart failure, and chronic obstructive pulmonary disease (COPD), are leading causes of avoidable hospitalizations. ${ }^{4}$

Chronic diseases are prolonged in duration and rarely completely cured. ${ }^{5}$ Understanding the impact of chronic disease on health care utilization requires modeling over extended periods of time. To perform this analysis, fixed reference points in disease progression must be identified. Reference points are required to inform clinical practice, identify trends in health care utilization, and to study phenomena in morbidity, such as "compression" (whereby morbidity is delayed while not similarly extending lifespan) ${ }^{6,7}$ and "expansion" (whereby death is delayed while not delaying onset of morbidity). ${ }^{8}$ Identifying such reference points are challenging.

Proximity to death has been used as fixed reference point and approximately a quarter of lifetime health care are consumed in the last year of life. ${ }^{9-11}$ However, these studies focus on patients in the last few years of life, rather than longer lived patients with chronic disease. Furthermore, death, as a reference point, precludes future health management at its identification.

To examine longitudinal demand, this paper describes an identifiable fixed reference point. This "cardinal event" is defined as a disease-specific diagnosis upon hospital admission, where such an event has not occurred in the previous 2 years. This definition identifies the first admission associated with a particular diagnosis in a potential series of admissions, within a reasonable period of time. 
Worldwide, there has been no previous study examining longitudinal hospital demand in the periods before and after hospital admissions for chronic disease. In this paper, longitudinal analysis is applied to hospital utilization around cardinal events in 3 of the most common chronic conditions: heart failure, type 2 diabetes, and COPD. The changes in utilization associated with such events, in terms of inpatient days and emergency department (ED) presentations, are compared with that seen in cardinal events in asthma, diabetic patients undergoing cataract procedures, and dialysis.

\section{METHODOLOGY}

\section{Ethics Statement}

Ethical approval was provided by the Department of Health Western Australia Human Research Ethics Committee and the University of Western Australia Human Research Ethics Committee. Informed consent was not sought and data were analyzed anonymously.

\section{Study Population}

Data were extracted from the Western Australia Data Collections, including the Hospital Morbidity, Emergency, and Mortality datasets. This dataset included all records from all public and private acute hospitals in Western Australia. ${ }^{12}$ All records (for any diagnosis) between 2002 and 2010 (inclusive) were obtained for patients who had at least 1 diagnosis (principal and/or secondary/alternative, at an ED presentation or admission to hospital within this time period) with one of the following International Statistical Classification of Diseases and Related Health Problems, Tenth Revision, Australian Modification (ICD-10-AM) codes: E9E14 (impaired glucose metabolism, including type 1 and type 2 diabetes), I50 (heart failure), and J40-J47 (including bronchitis, COPD, and asthma). This dataset defined the population of patients from which cardinal events would be extracted.

Records for individuals identified as Aboriginal or Torres Strait Islander $3.7 \%$ of the Western Australian population ${ }^{13}$ ) were excluded from the analysis due to statistically significantly different distributions of age at diagnosis, rate of hospitalization, and mortality (data not shown).

\section{Definition of Cardinal Event}

"Cardinal events" were defined as the first day of an admission to hospital (not including ED visits that did not result in an admission) associated with a particular principal (and not additional) diagnosis code, where the same principal diagnosis was not associated with an admission by that patient in the previous 2 years. This excluded all admissions in 2002 and 2003 as admissions in these years could not be identified using these criteria. This resulted in a "rolling" 2year clearance period. Cardinal events after 2008 were excluded to allow sufficient time (2009 and 2010) for subsequent events to be recorded within the Western Australia Data Collections, as records enter the database after discharge. A minority of individuals had multiple events ( 2 and, rarely, 3 such events with the same diagnosis) occurring in the 5-year study period (see the Results section).
Cardinal events were identified using the ICD-10-AM diagnosis codes E11 ("type 2 diabetes mellitus," excluding E11.39 "type 2 diabetes mellitus with other specified ophthalmic complication," hereafter referred to as "type 2 diabetes"), I50 (heart failure), J44 ("other chronic obstructive pulmonary disease," ie, excluding emphysema, hereafter "COPD"), J45 or J46 ("asthma" and "status asthmaticus," hereafter "asthma"), and Z49 ("care involving dialysis," hereafter "dialysis"). Approximately $60 \%$ of all admissions coded under a principal diagnosis of E11 were E11.39 (type 2 diabetes mellitus with other specified ophthalmic complication) and $97 \%$ of these had procedure codes related to cataract operations (data not shown). These were designated "cataract with diabetes."

\section{Statistical Analysis}

A generalized linear mixed effects (logistic) model ${ }^{14}$ was used to analyze the proportion of diagnosis-specific cardinal events in each study year (2004-2008), including presence/absence of cardinal event as the dependent variable, year as the independent variable, and random effects for the unique subject identifier. This provides a correlation structure to account for those individuals who had 1, 2, or 3 cardinal events during the study period (see Supplemental Digital Content 1, http://links.lww.com/MLR/A767). Note that few patients had 3 events and the correlation structure in this group may be imprecise.

Patient data before and after a cardinal event were aligned using the day of the cardinal event as time zero. After adjusting for entry into the monitored data period, loss to follow-up, and death, the number of person-days contributing to the denominator value (n) changed with time. The dataset is therefore both right and left censored, that is, patients may not have data for the full 6 years before or 4 years after the cardinal event (see Table, Supplemental Digital Content 2, http://links.lww.com/MLR/A768). Admissions to hospital (including transfers) were linked to form an inpatient history (while retaining inpatient days actually used), with leave days (ie, absence from hospital, occurring in $<2 \%$ of admissions) being excluded. Similarly, an ED presentation history was prepared. Daily hospital utilization was calculated as the number ED presentations or inpatient days (calculated and presented separately in the Results section) in the numerator divided by person-days in the denominator on a given day.

Hospitalizations with a principal diagnosis code of dialysis were removed from the analysis to avoid bias from such events. Same-day admissions for dialysis was associated with approximately $40 \%$ of all admissions in our study population, but were associated with only $0.5 \%$ of all patients (data not shown). The exception was that same-day admissions for dialysis were included where the cardinal event diagnosis was itself dialysis. Note that dialysis cardinal events only include patients who have been diagnosed with a chronic disease.

Nonparametric regression was used, based on locally weighted polynomial regression ${ }^{15}$ (LOESS), to provide an accurate estimate of mean hospital utilization (as measured by either ED presentation or inpatient days), excluding a 
2-week time window prior and after the cardinal event. This allowed $95 \%$ confidence intervals (CI) to be simply generated using bootstrap techniques. LOESS combines the simplicity of least squares regression with the flexibility of nonlinear regression. Simple models are fitted to localized subsets of the data to build a curve that describes the variation in the data. This method does not require a global function or any assumption of form to fit a model to the data (see Supplemental Digital Content 3, http://links.lww.com/ MLR/A769).

Estimates were displayed for the period up to 31 days before, and for the period beginning 31 days following, the cardinal event. The loess function (stats package in the $\mathrm{R}$ Statistical Graphics version 2.15.3 software ${ }^{16}$ ) was used to fit a kernel-weighted, polynomial regression with a second-order polynomial, with a Gaussian kernel with a span of 1.4 and weights equal to the denominator value on each day. The span was chosen by eye to balance bias (an over-smoothed curve) against variance (a curve that "chases the points"). Ninety-five percent confidence limits were calculated using a bootstrap function (bootobject and boot.ci functions from the boot package in $\mathrm{R}^{17}$ ). A total of 5000 bootstrap replicates were used (sampling with replacement) and $95 \%$ confidence limits were obtained using the bootstrap percentile method. ${ }^{18}$

Total number of ED presentations and inpatient days were separately calculated for 3 periods relative to a cardinal event: "around event," which is between -30 and +30 days; "before event," between -31 days and -6 years; and "after event," between +31 days and +4 years. Ninety-five percent CI for the hospitalization rates were calculated using the bootstrap percentile interval, using 1000 replicates sampled with replacement. ${ }^{18}$

\section{RESULTS}

\section{Cardinal Events}

The total number of cardinal events examined is 47,443 . Overwhelmingly, only a single cardinal event for each diagnosis type can be identified for any individual within the time period examined, with the percentage of events being the second for an individual with, for example, heart failure, type 2 diabetes, and COPD being 3, 4, and 6, respectively (Table 1). Less than 5 individuals experience a third cardinal event. Table 1 shows the age and sex distributions of patients with cardinal events.

Figure 1 shows number of admissions each year, with each bar divided into admissions identified as cardinal events and those that are not. Dialysis admissions are not shown, due to scale. Yearly noncardinal/cardinal admissions for dialysis from 2004 to 2008 are $49,120 / 154,50,768 / 183$, $50,684 / 167,52,474 / 187$, and 56,968/197, respectively. The percentage of the all admissions for the diagnoses examined that are identifiable as cardinal events from 2004 to 2008 for heart failure, type 2 diabetes, COPD, cataract with diabetes, asthma, and dialysis were $60 \%, 58 \%, 44 \%, 70 \%, 68 \%$, and $0.3 \%$, respectively.

Criteria for cardinal events are time unbiased (ie, the likelihood of a cardinal event being identified does not change over time), therefore changes in cardinal event rates

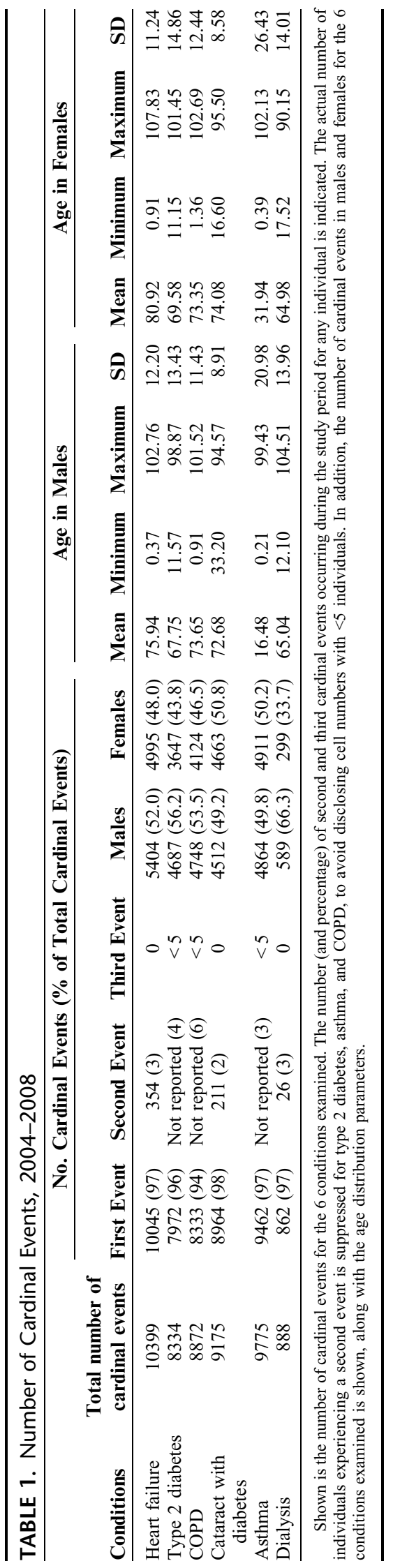




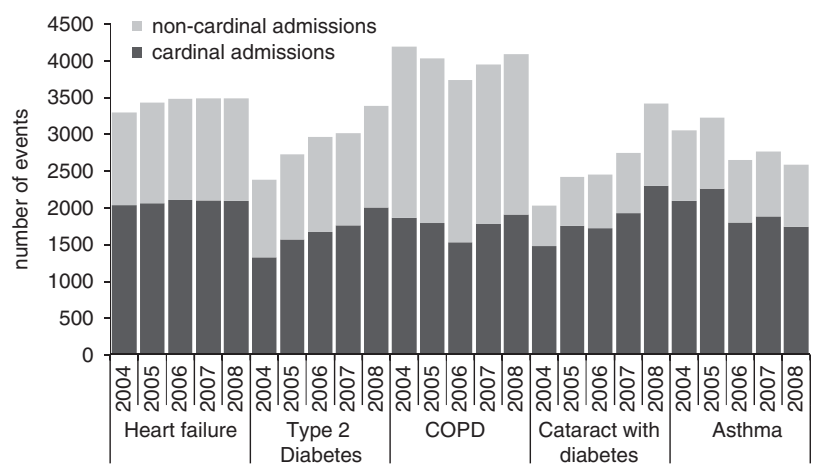

FIGURE 1. Cardinal and noncardinal admissions over time. Shown is the number of admissions in each year from 2004 to 2008 , for each of the conditions examined. The admissions are separated into those identifiable as cardinal events, and those that are not.

can be analyzed. Type 2 diabetes cardinal events increase $12 \%$ per year $(95 \% \mathrm{CI}, 10 \%-14 \%, P<0.00001)$. Cataract with diabetes cardinal events increase $13 \%$ per year $(95 \% \mathrm{CI}$, $11 \%-15 \%, P<0.00001)$. Dialysis cardinal events increase $7 \%$ per year $(95 \% \mathrm{CI}, 1 \%-12 \%, P=0.017)$. Asthma cardinal events decrease by $7 \%$ per year $(95 \%$ CI, 5\%-9\%, $P<0.00001)$. No significant trends in heart failure or COPD events are observed.

\section{Longitudinal Analysis}

The longitudinal hospital utilization (defined as inpatient days or ED presentations) associated with cardinal events are shown in Figures 2 and 3. The period examined was from 6 years before the cardinal event, to 4 years following. Estimates are displayed for the period up to 31 days before the cardinal event, and for the period beginning 31 days following the cardinal event. Multiple cardinal events for the same individual are included in this analysis. Excluding such events did not significantly alter the analysis shown below (data not shown). The n-values, estimates, and confidence bands at $-6,-4,-2,+2$, and +4 years relative to the cardinal event are detailed in Table (Supplemental Digital Content 2, http://links.lww.com/MLR/A768).

As shown in Figures $2 \mathrm{~A}-\mathrm{C}$, the rate of inpatient days in the years around a cardinal event in heart failure, type 2 diabetes, and COPD are strikingly similar. Six years before the cardinal event, inpatient days were 3-5 days/year. This increases over the next 4 years, more rapidly in the 2 years before the cardinal event. After the event, inpatient days decrease rapidly in the first year, before reaching new levels between approximately 9 and 14 days/year at 2 years following the cardinal event, and between approximately 14 and 16 days/year at 4 years following the cardinal event. Excluding 1 year immediately before and after the event, rates of inpatient days are consistently 2-3 times higher after the event when compared with before.

In contrast, inpatient days/year in "cataract with diabetes" cardinal events increase steadily across the 10-year period, from approximately 2-7 days/year (Fig. 2D). Inpatient days/year around asthma cardinal events follow an- other trajectory, increasing from approximately 1 day/year at 6 years prior and plateauing at approximately 2 days/year at 3 years before the cardinal event. This rate is maintained until after the cardinal event, where rates immediately following the cardinal event are approximately 3 days/year, before reducing to approximately 2 days/year at 4 years following the cardinal event, that is, the same rates observed 3 years before the cardinal event (Fig. 2E). Inpatient rates around dialysis cardinal events increase from approximately 5 days/year in the years before the cardinal event, to approximately 90 days/year in the years following the cardinal event (Fig. 2F). As noted in the methodology, dialysis admission events are included in the analysis of dialysis cardinal events, but not in other cardinal event analyses.

In cardinal events associated with heart failure, type 2 diabetes, and COPD (Figs. 3A-C), the rate of ED presentations per year (presentations/year) increases from approximately 0.5 presentations/year ( 6 y pre-event) to $0.6-0.8$ presentations/year ( $2 \mathrm{y}$ pre-event). Rates peak around the cardinal event, before returning to levels of between 1 and 1.5 ED presentations/year in the years following the cardinal event. These levels remain above 1 presentation/year until at least 4 years after the cardinal event. Excluding 1 year immediately before and after the event, rates of ED presentations were consistently 2-3 times higher after the event when compared with before.

In contrast, ED presentations/year in cataract with diabetes cardinal events increase steadily across the 10-year period, from approximately 0.25 ED presentations/year at 6 years prior, to $0.6 \mathrm{ED}$ presentations/year (Fig. 3D). ED presentations/year around asthma cardinal events increase from approximately $0.5 \mathrm{ED}$ presentations/year at 6 years prior, increasing to $0.8 \mathrm{ED}$ presentations/year 2 years before the cardinal event. The rate peaks around the cardinal event, before returning to a similar rate 4 years after the cardinal event (Fig. 3E). Inpatient rates around dialysis cardinal events increase from approximately 0.5 ED presentations/ year at 6 years prior, to 0.9 ED presentations/year 2 years before the cardinal event. Following a peak around the cardinal event, these rates then drop to approximately $1.4 \mathrm{ED}$ presentations/year after 4 years (Fig. 3F).

\section{Total Utilization Across Periods}

The utilization patterns described above excludes utilization in a period around the cardinal event, from 30 days before to 30 days after. To examine this period, total number of ED presentations and inpatient days were separately calculated for 3 periods relative to a cardinal event: "around event," "before event," and "after event," and shown in Figure 4 (see Table, Supplemental Digital Content 4, http://links.lww.com/MLR/A770). As shown, hospital utilization in the period around the cardinal event for heart failure, type 2 diabetes, COPD, and asthma, represents only $10 \%-15 \%$ of the total demand for inpatient days and for ED presentations across the 10 -year period examined.

\section{DISCUSSION}

Cardinal events describe a fixed reference point in disease progression, enabling examination of trends in 
A

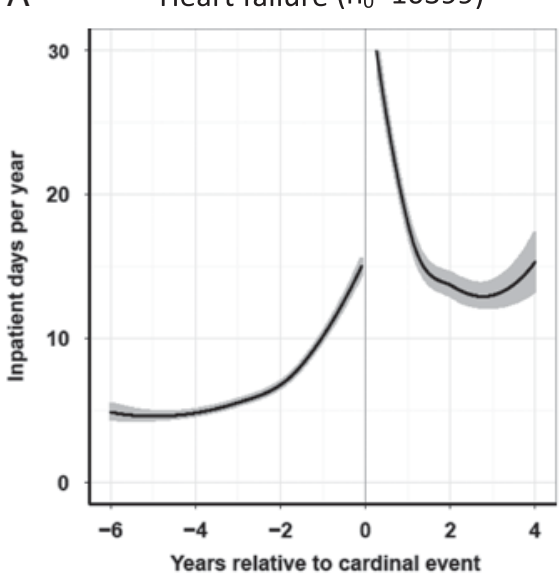

D Cataract with diabetes $\left(n_{0}=9175\right)$

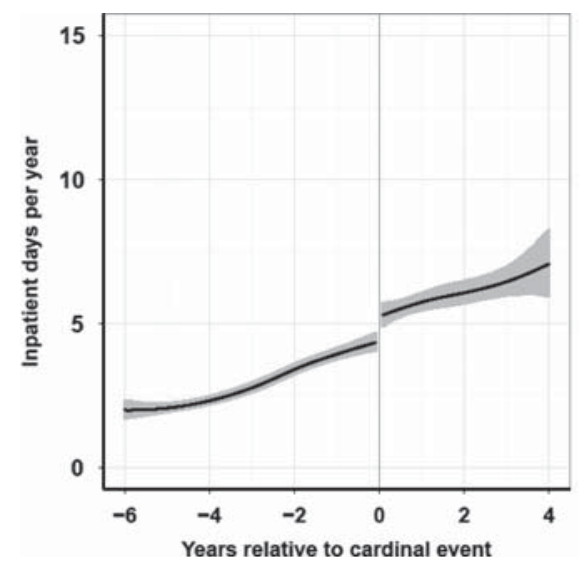

B

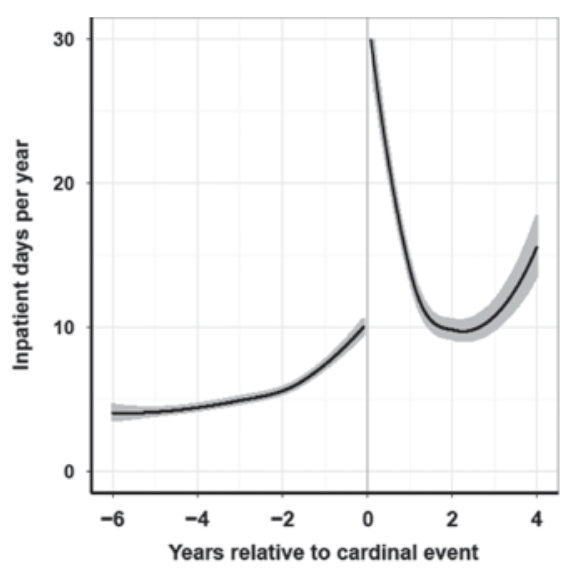

E

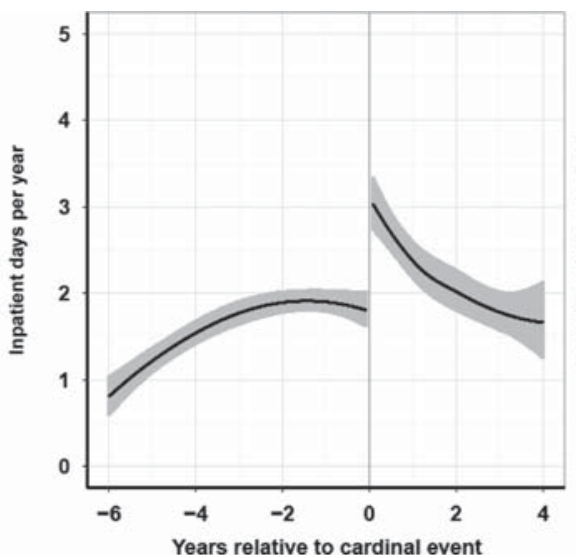

C

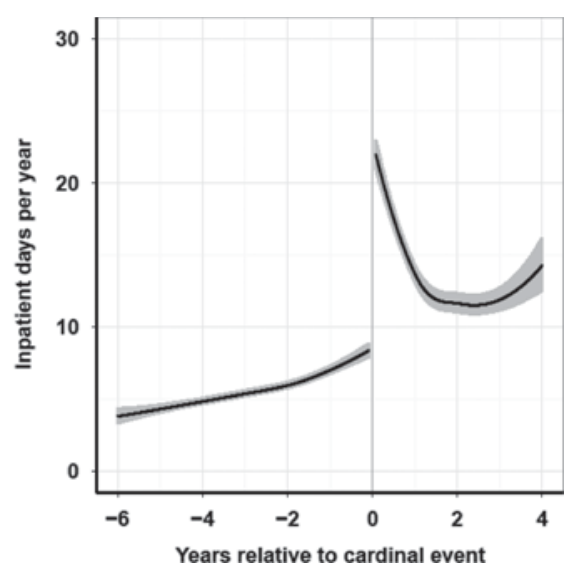

$\mathrm{F}$

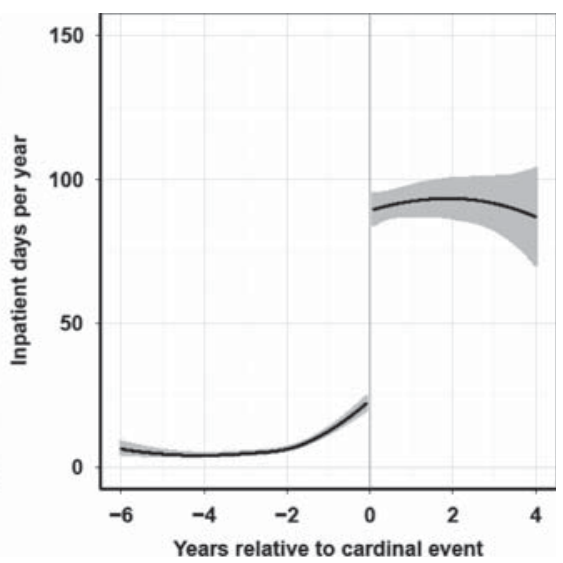

FIGURE 2. A-F, Longitudinal analysis of inpatient days per year around cardinal events. Shown is the mean inpatient days per year (and 95\% confidence bands) in the 6 years before and 4 years following a cardinal event for each of the 6 conditions examined. The number of cardinal events at time zero (marked with a dotted line) in each analysis is indicated $\left(n_{0}\right)$.

hospital utilization over time. We demonstrate that for 3 of the most common chronic diseases, the cardinal event heralds a marked and sustained change in days in hospital and ED presentations. The 2-year clearance period was defined to maximize the lead-in and follow-up data available, while minimizing multiple events. Different clearance periods before the cardinal event, for example, 4 years rather than 2 , did not significantly change our findings (data not shown) but reduced the follow-up period available for analysis.

Alternatively, date of "first" diagnosis with disease may be used as a fixed reference point. However, the identification of a "first event" is not straightforward. "First events" are subject to "prevalent pool effects," where the actual first event may occur outside the period under examination. A clearance period at the start of a study can reduce this effect, ${ }^{19,20}$ but this results in early "first-time" events in the data collection period having a shorter clearance period than later "first-time" events, that is, nonequivalent time-biased reference points. "Back-casting" methods ${ }^{21}$ are unsuitable in identifying reference points, as these allow for adjustment of incidence rates to remove the prevalent pool effect, but do not allow for identification of individual events. The definition used here for cardinal events overcomes these issues.

Cardinal events represent $40 \%-60 \%$ of the chronic disease admissions examined. Type 2 diabetes-related cardinal events are increasing, consistent with worldwide trends. ${ }^{22}$ No changes in the Australian coding standards for ICD-10-AM adequately explain this consistent trend. However, a limitation of this methodology is potential biases introduced by inconsistent and changing coding practices over time.

Following a cardinal event for type 2 diabetes mellitus, COPD, heart failure, and dialysis, there is a marked increase in all-cause demand that is sustained for the following 4 years. This change seems to be relatively stable for years after the event and is analogous to a "ratchet effect," that is, a change relatively resistant to reversal. This ratchet effect is consistent across the 3 most common chronic diseases and is not seen in following cardinal events for asthma or in cataract with diabetes.

We excluded 60 days around the cardinal event to produce the smoothed utilization curves. It could be argued that most hospital utilization would occur around the 
A

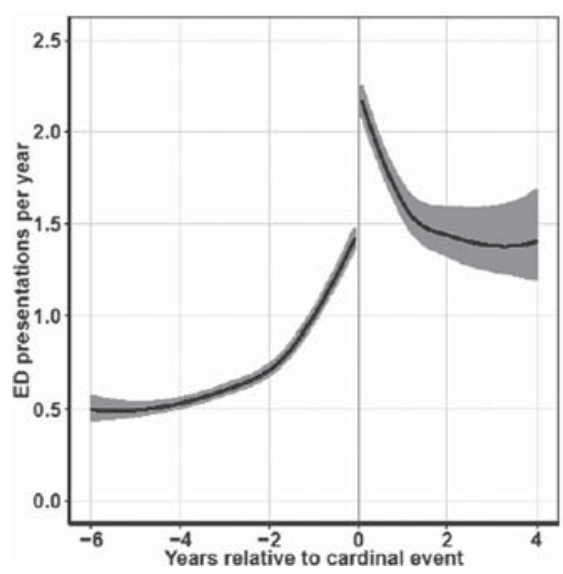

D Cataract with diabetes $\left(n_{0}=9175\right)$

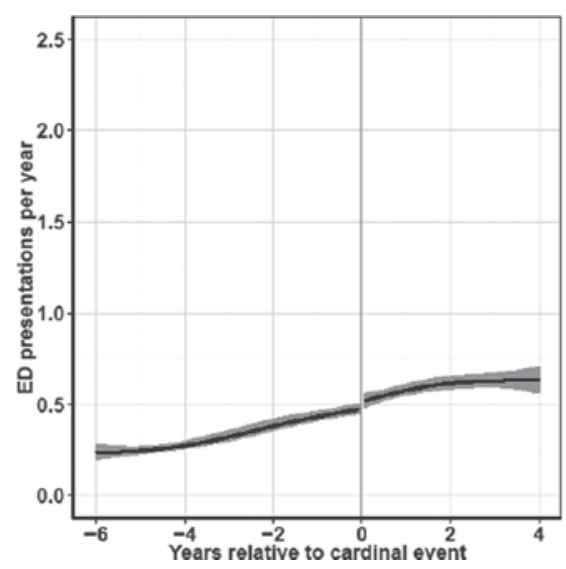

B

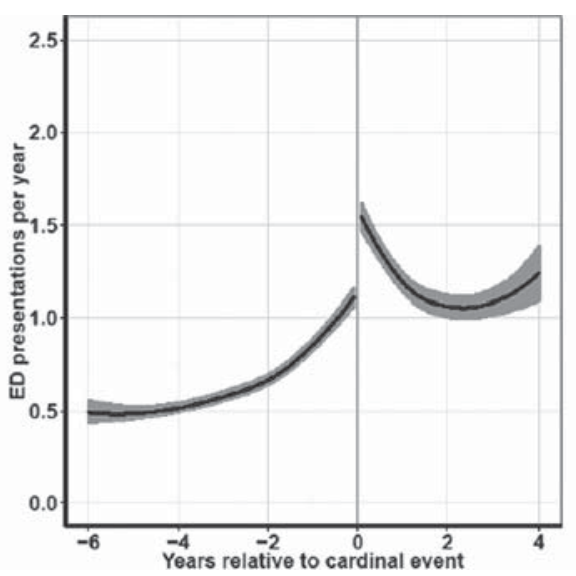

E

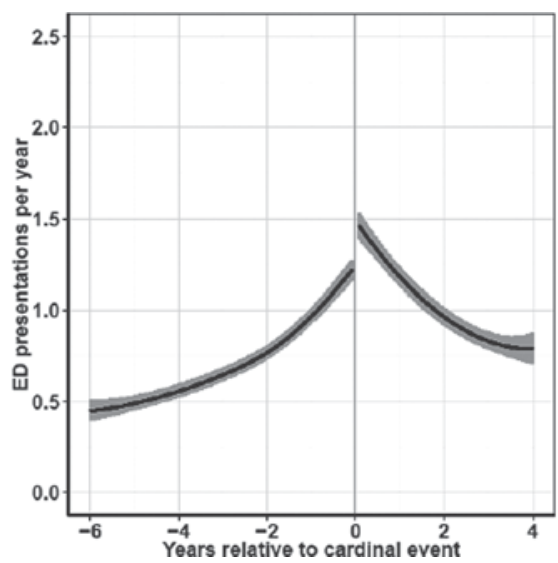

C

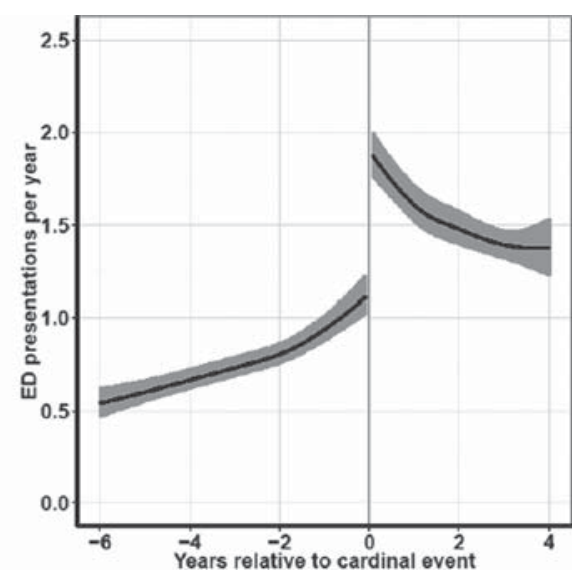

$\mathrm{F} \quad$ Dialysis $\left(\mathrm{n}_{0}=888\right)$

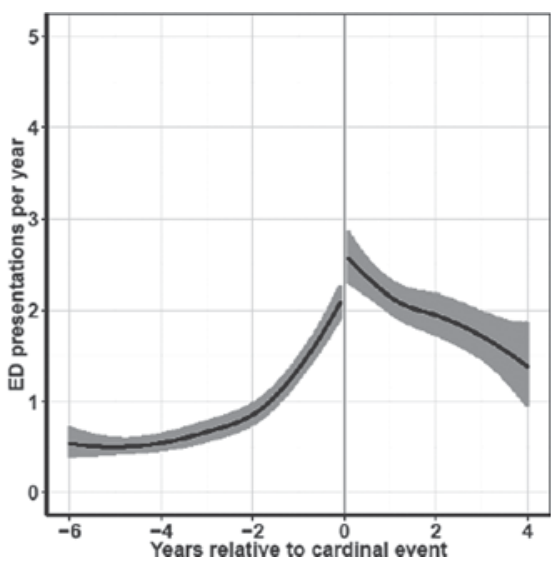

FIGURE 3. A-F, Longitudinal analysis of emergency department presentations per year around cardinal events. Shown is the mean emergency department presentations per year (and 95\% confidence bands) in the 6 years before and 4 years following a cardinal event for each of the 6 conditions examined. The number of cardinal events at time zero (marked with a dotted line) in each analysis is indicated $\left(n_{0}\right)$.

cardinal event, rather than outside this period. However, $85 \%-90 \%$ of the demand occurs outside of this 60 -day period.

Other studies have examined health service utilization following a diagnosis of chronic disease,${ }^{23-26}$ or in the period preceding a diagnosis. ${ }^{27}$ However, these studies did not examine the change (comparing pre and post) in demand associated with such a diagnosis. It is noteworthy that health service utilization before and after a diagnosis of type 2 diabetes in primary care has been examined, and no ratchet effect was observed in that setting. ${ }^{28}$ This is the first time that all-cause inpatient days and ED presentations both before and after a hospital diagnosis with chronic disease has been examined. Furthermore, previous studies have not used the robust statistical methods or large datasets as the study presented here. ${ }^{23-26,29,30}$

The ratchet in hospital utilization following a cardinal event in chronic disease is not expected. Rather, it may be anticipated that an admission occurs during an increasing pattern of hospital utilization. For example, it has been observed that hospital admission rates steadily rise around the time of a diagnosis of type 2 diabetes in general practice. ${ }^{28}$ In a planned admission to hospital for a routine procedure (ie, a cataract operation for a diabetic patient), such gradual increases in all-cause hospital admissions and ED presentations were observed. Alternatively, an admission may reflect an exacerbation of chronic disease, resulting in a rise in hospital use which subsides following treatment, as observed in the case of asthma. Where patients undergo a dramatic alteration in condition or clinical care, a dramatic and sustained increase in utilization may result. For patients commencing dialysis, this reflects an expected change, where usual treatment requires dialysis 2-3 times per week and ED presentations are known to increase. ${ }^{31}$ Unexpectedly, this pattern was observed in type 2 diabetes, heart failure, and COPD. Some ED presentations after these 3 chronic disease cardinal events would be due to patients entering dialysis. However, the small number of patients entering dialysis, and the relative increase in ED presentations after dialysis initiation, demonstrates that dialysis is a minor contributor to this increase.

Factors driving the ratchet effect may include changes in clinician and patient behavior, and changes in severity of 

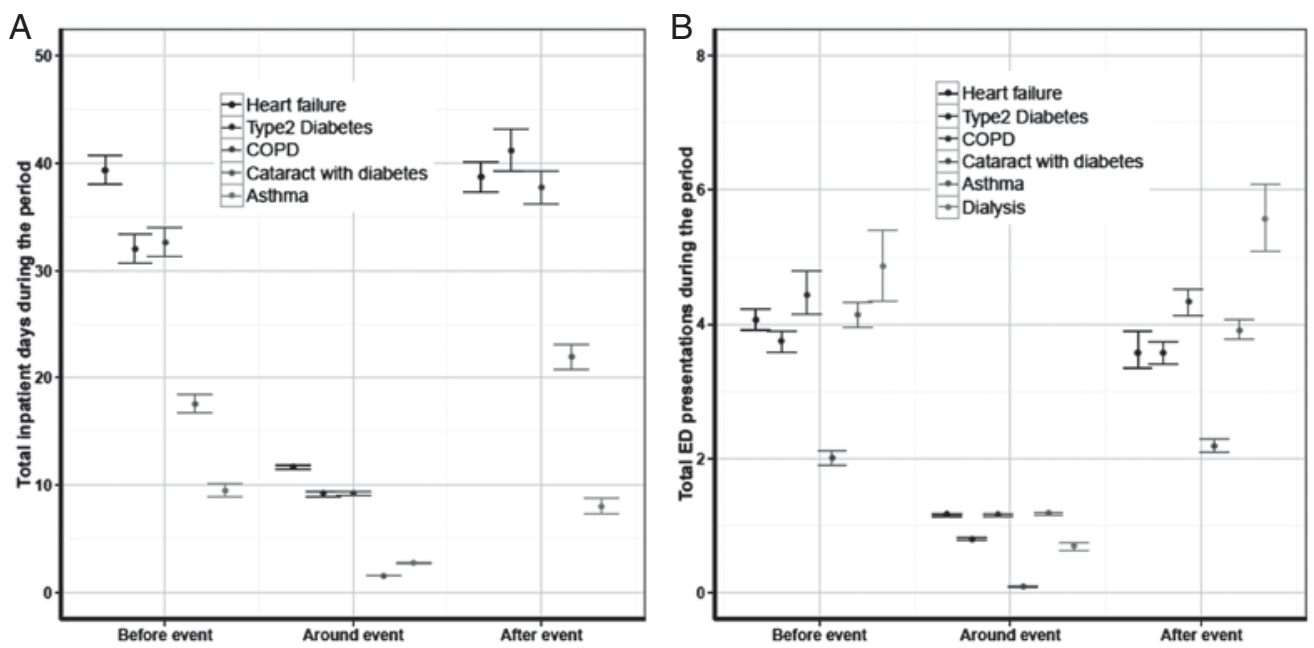

FIGURE 4. Total hospital utilization in the periods before, around, and after cardinal events. A, Shown are the mean (and $95 \%$ confidence interval) of total inpatient days associated with a cardinal event, in the 6 years before the event (up to $31 \mathrm{~d}$ before the event), in the period around the event (from day -30 to day +30$)$, and in the 4 years after the event (from $+31 \mathrm{~d}$ onward). Note that dialysis inpatient days are not indicated due to being off scale. B, Shown are the mean (and 95\% confidence interval) of total emergency department presentations associated with a cardinal event, in the 6 years before to the event (up to $31 \mathrm{~d}$ before the event), in the period around the event (from day -30 to day +30 ), and in the 4 years after the event (from $+31 \mathrm{~d}$ onward).

underlying disease. There may be increased planned care following an admission. However, planned care alone does not account for the increases in all-cause ED presentations following the cardinal event. Clinicians may encourage patients to re-present to ED with exacerbations. Individuals or their carers may make new choices in the appropriate site for management of their disease. Cardinal events may also be a transition across a threshold in disease progression. Finally, increased demand may be associated with patients approaching death.

In this paper, records for individuals who were identified at least once as Aboriginal or Torres Strait Islander were excluded from the analysis due to statistically significantly different distributions of age at diagnosis, rate of hospitalization, and mortality. This does not weaken the generalizability of our findings outside of Australia, as Aboriginal and Torres Strait Islanders are a group unique to Australia. Current analysis is focused on outlining the differences in hospital demand in this group (manuscript in preparation). ${ }^{32}$ Furthermore, we have specifically focused on cardinal events as defined by a diagnosis in hospital, as such events are well defined and of importance to the providers of hospital-based services. We have not examined cardinal events associated with other identifiable reference points in disease progression (eg, a diagnosis in primary care). Furthermore, we have not examined changes in outpatient utilization, although such changes may be significant.

This paper identifies dramatic and sustained increases in all-cause inpatient days and ED presentations associated with specific chronic disease events. This ratchet effect associated with cardinal events is important in understanding the impact of chronic disease on hospital demand. Events that herald such a marked transition in health service demand have not been previously described. Better primary care with a focus on chronic disease may delay such cardinal events, and programs may be identified that manage more appropriate care following cardinal events. The identification of such a reference point in care allows these possibilities to be examined.

\section{REFERENCES}

1. Beaglehole R, Bonita R, Horton R, et al. Priority actions for the noncommunicable disease crisis. Lancet. 2011;377:1438-1447.

2. Piot P, Ebrahim S. Prevention and control of chronic diseases. BMJ. 2010;341:c4865-c4865.

3. Anderson G, Horvath J. The growing burden of chronic disease in America. Public Health Rep. 2004;119:263-270.

4. Glover J, Page A, Ambrose S, et al. Atlas of Avoidable Hospitalisations in Australia: Ambulatory Care-Sensitive Conditions. Cat. No. HSE 49. Canberra: Australian Institute of Health and Welfare (AIHW); 2007.

5. Australian Institute of Health and Welfare (AIHW). Risk Factors Contributing to Chronic Disease. Cat No. PHE 157. Canberra: AIHW; 2012.

6. Fries JF. Aging, natural death, and the compression of morbidity. N Engl J Med. 1980;303:130-135.

7. Fries J. Frailty, heart disease, and stroke: the compression of morbidity paradigm. Am J Prev Med. 2005;29(suppl 1):164-168.

8. Olshansky SJ, Rudberg MA, Carnes BA, et al. Trading off longer life for worsening health: The expansion of morbidity hypothesis. J Aging Health. 1991;3:194-216.

9. Zweifel P, Felder S, Meiers M. Ageing of population and health care expenditure: a red herring? Health Econ. 1999;8:485-496.

10. Seshamani M, Gray A. A longitudinal study of the effects of age and time to death on hospital costs. J Health Econ. 2004;23:217-235.

11. Seshamani M, Gray A. Ageing and health-care expenditure: the red herring argument revisited. Health Econ. 2004;13:303-314.

12. Holman CD, Bass AJ, Rouse IL, et al. Population-based linkage of health records in Western Australia: development of a health services research linked database. Aust N Z J Public Health. 1999;23:453-459.

13. Australian Bureau of Statistics (ABS). Australian Historical Population Statistics, 2008. Cat. No. 3105.0.65.001. Canberra: ABS; 2008.

14. Pinheiro J, Bates D. Mixed Effects Models in S and S-Plus. 1st ed. New York: Springer-Verlag; 2000.

15. Cleveland WS, Grosse E, Shyu WM. Local regression models. In: Chambers JM, Hastie TJ, eds. Statistical Models in S. New York: Chapman \& Hall; 1992. 
16. R Development Core Team. R: A Language and Environment for Statistical Computing. Vienna, Austria: Foundation for Statistical Computing; 2013. Available at: http://www.R-project.org/. Accessed October 16, 2013.

17. Canty A, Ripley B. Bootstrap Functions. CRAN, 2013. Available at: http://CRAN.R-project.org/. Accessed October 17, 2013

18. Davison A, Hinkley D. Bootstrap Methods and Their Application. Cambridge, NY: Cambridge University Press; 1997.

19. Huff L, Bogdan G, Burke $\mathrm{K}$, et al. Using hospital discharge data for disease surveillance. Public Health Rep. 1996;111: 78-81.

20. Brameld K, Holman D, Thomas M, et al. Use of a state data bank to measure incidence and prevalence of a chronic disease: end-stage renal failure. Am J Kidney Dis. 1999;34:1033-1039.

21. Brameld K, Holman CDAJ, Lawrence D, et al. Improved methods for estimating incidence from linked hospital morbidity data. Int $J$ Epidemiol. 2003;32:617-624.

22. Danaei G, Finucane MM, Lu Y, et al. National, regional, and global trends in fasting plasma glucose and diabetes prevalence since 1980: systematic analysis of health examination surveys and epidemiological studies with 370 country-years and 2.7 million participants. Lancet. 2011;378:31-40

23. Brown JB, Nichols GA, Glauber HS, et al. Type 2 diabetes: incremental medical care costs during the first 8 years after diagnosis. Diabetes Care. 1999;22:1116-1124.
24. Migliaccio-Walle K, Caro JJ, Ishak KJ, et al. Costs and medical care consequences associated with the diagnosis of peripheral arterial disease. Pharmacoeconomics. 2005;23:733-742.

25. Geelhoed EA, Brameld KJ, Holman CDJ, et al. Readmission and survival following hospitalization for chronic obstructive pulmonary disease: long-term trends. Intern Med J. 2007;37:87-94.

26. Wahle C, Adamopoulos C, Ekundayo OJ, et al. A propensity-matched study of outcomes of chronic heart failure (HF) in younger and older adults. Arch Gerontol Geriatr. 2009;49:165-171.

27. Nichols GA, Glauber HS, Brown JB. Type 2 diabetes: Incremental medical care costs during the 8 years preceding diagnosis. Diabetes Care. 2000;23:1654-1659.

28. Brameld K, Ward A, Gavin A, et al. Health outcomes in people with type 2 diabetes. A record linkage study. Aust Fam Physician. 2002;31:775-882.

29. Bohl A, Fishman P, Ciol M, et al. A longitudinal analysis of total 3-year healthcare costs for older adults who experience a fall requiring medical care. J Am Geriatr Soc. 2010;58:853-860.

30. Duclos A, Couray Targe S, Randrianasolo M, et al. Burden of hip fracture on inpatient care: a before and after population-based study. Osteoporosis Int. 2010;21:1493-1501.

31. Loran MJ, McErlean M, Eisele G, et al. The emergency department care of hemodialysis patients. Clin Nephrol. 2002;57:439-443.

32. Tenneti R, Marsh J, Pearson G, et al. Hospital utilisation in Aboriginal and non-Aboriginal patients with chronic disease. Manuscript in preparation. 2014 